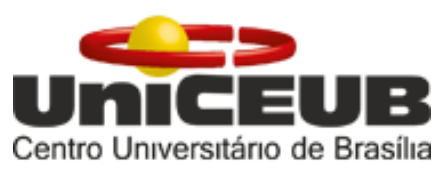

CENTRO UNIVERSITÁRIO DE BRASÍLIA - UniCEUB

PROGRAMA DE INICIAÇÃO CIENTÍFICA

JÚLIA MILHOMEM MOSQUÉRA

AMANDA RIBEIRO ALVES

IDENTIFICAÇÃO DE FATORES ASSOCIADOS A MORBIMORTALIDADE DE IDOSOS COM FRATURAS DE QUADRIL POR FRAGILIDADE ÓSSEA EM INTERNAÇÃO HOSPITALAR.

BRASÍLIA

2018 


\section{Uित्र \\ Centro Unıversitárıo de Brasílıa}

JÚLIA MILHOMEM MOSQUÉRA

AMANDA RIBEIRO ALVES

IDENTIFICAÇÃO DE FATORES ASSOCIADOS A MORBIMORTALIDADE DE IDOSOS COM FRATURAS DE QUADRIL POR FRAGILIDADE ÓSSEA EM INTERNAÇÃO HOSPITALAR.

Relatório final de pesquisa de Iniciação

Científica apresentado à Assessoria de Pós-

Graduação e Pesquisa.

Orientação: Viviane Cristina Uliana Peterle

BRASÍLIA 


\title{
IDENTIFICAÇÃO DE FATORES ASSOCIADOS A MORBIMORTALIDADE DE IDOSOS COM FRATURAS DE QUADRIL POR FRAGILIDADE ÓSSEA EM INTERNAÇÃO HOSPITALAR.
}

\author{
Júlia Milhomem Mosquéra- UniCEUB, PIC Institucional, aluno bolsista \\ j.milhomemosquera@sempreceub.com
}

\author{
Amanda Ribeiro Alves- UniCEUB, PIC institucional, aluno voluntário \\ amanda.ribeiro@sempreceub.com
}

\author{
Viviane Cristina Uliana Peterle- UniCEUB, professor orientador \\ viviane.peterle@ceub.edu.br
}

INTRODUÇÃO: A osteoporose constitui um importante problema de saúde pública devido a sua associação com fraturas relacionadas com a idade, principalmente as do fêmur proximal. As causas para a elevada mortalidade em pacientes idosos com fratura da extremidade proximal do fêmur foram bem investigadas nos países desenvolvidos; no entanto, pouco se sabe sobre este assunto nos países em desenvolvimento. $\mathrm{O}$ objetivo do trabalho foi identificar fatores de morbidade e mortalidade intra-hospitalar de pacientes idosos com fragilidade óssea com fratura de quadril, bem como os fatores clínicos associados ao evento. METODOLOGIA: Estudo de coorte histórica realizado a partir da coleta de dados de prontuários de pacientes internados em Serviço Público de Ortopedia do Distrito Federal. Para o estudo, considerou-se os pacientes admitidos entre janeiro de 2010 e janeiro de 2017. A análise bivariada para testar a associação com o desfecho (mortalidade) realizada através do teste qui-quadrado e a comparação entre grupos (óbito versus sobrevivência) através do teste de Mann-Whitney. Valores de p<0,05 foram considerados estatisticamente significativos. A fim de avaliar quais variáveis impactaram no desfecho óbito e qual a razão de chance dos preditores selecionados, foi construído um modelo de regressão logística através do método Stepwis. As análises de sobrevivência foram realizadas através da regressão logística de COX e da curvas de Kaplan-Meier. RESULTADOS: Foram identificados 349 pacientes que atenderam aos critérios de inclusão e

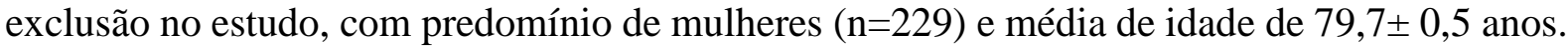
Observou-se que 76 pacientes $(21,8 \%)$ tiveram a mortalidade como desfecho e na análise de regressão logística ajustado para idade e sexo [IC95\%], as variáveis que explicaram a mortalidade pelo modelo $(\mathrm{p}<0,005)$ foram presença de infecção [OR=5,7 $(\mathrm{p} 0,026)]$, Infecção Respiratória $[\mathrm{OR}=7,38(\mathrm{p} 0,006)]$, tempo em UTI $[\mathrm{OR}=1,10(\mathrm{p} 0,004)]$ e Hipertensão Arterial $[\mathrm{OR}=4,05$ (p0,003)]. A avaliação perioperatória realizada (Detsky, 1997), também demostra associação com maior mortalidade entre os pacientes classificados como III (RR 10,6) [n=51; $68 \%$ 0,000). O tempo de pós-operatório médio foi de 19,6 dias. Desde a internação do paciente, o tempo médio de sobrevida foi de 62,7 dias [IC95\% 47,8-77,6]. Considerando apenas os pacientes que realizaram cirurgia e o tempo pós operatório, verificou-se que o tempo em média 
de sobrevida foi de 39,2 dias [IC95\% 26,34-53,33;]. DISCUSSÃO/CONCLUSÃO: O tratamento de escolha para a maioria dos pacientes com fratura de fêmur é o cirúrgico. Sabe-se que quanto maior o número de comorbidades no paciente idoso fraturado, maior é a chance de evolução para óbito. Esses dados impactam diretamente na decisão cirúrgica, tempo de intervenção e prognóstico, como exemplo, a alta precoce que pode reduzir o tempo de exposição a agentes infecciosos intra-hospitalares.

Palavras-Chave: Idoso. Mortalidade. Fratura De Quadril. Osteoporose. 


\section{SUMÁRIO}

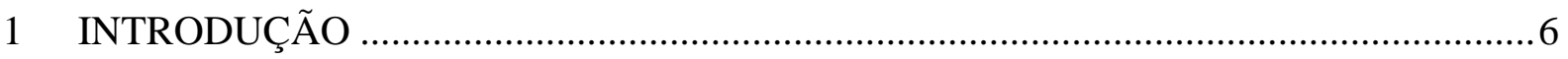

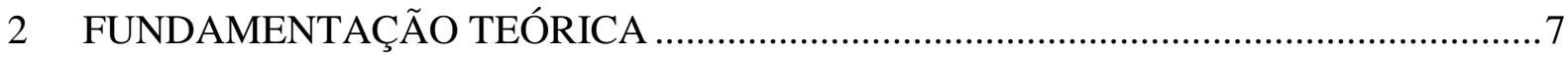

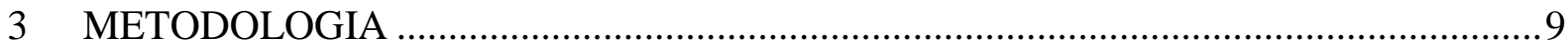

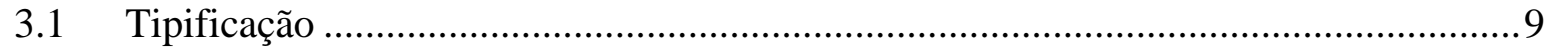

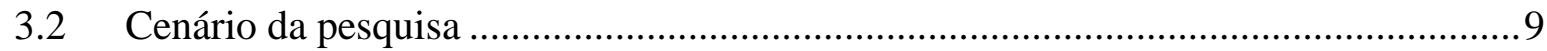

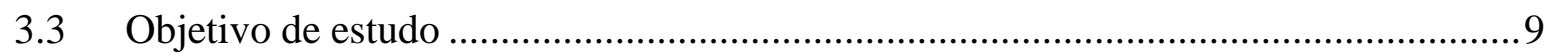

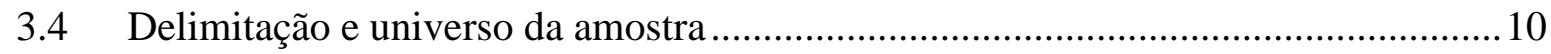

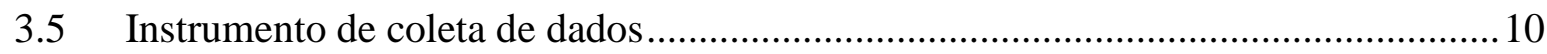

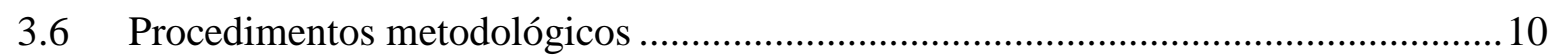

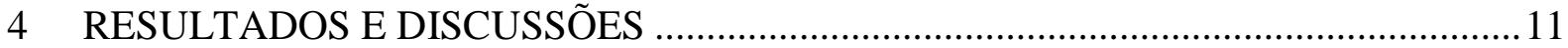

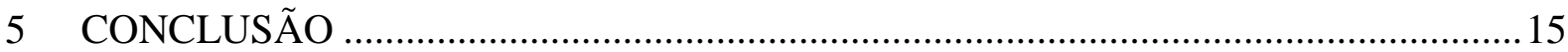

6 REFEREANCIAS: 


\section{INTRODUÇÃO}

Osteoporose é definida como uma doença óssea caracterizada pelo comprometimento da resistência óssea que predispõe a um aumento do risco de fratura. A fratura por fragilidade óssea é a maior expressão clínica dessa doença. Fratura por fragilidade é definida pela Organização Mundial de Saúde como "uma fratura causada por um trauma que seria insuficiente para fraturar um osso normal, resultado de uma redução da resistência compressiva ou torsional"“. Do ponto de vista clínico poderia ser definida como uma fratura que ocorre como o resultado de um trauma mínimo, como queda da própria altura ou menor ou por trauma não identificado. As fraturas por fragilidade típicas incluem vértebras, fêmur proximal (quadril), rádio distal e úmero proximal (STOLNICK, 2016).

A queda é o acidente que ocorre com maior frequência no idoso, sendo suas complicações a principal causa de morte naqueles com mais de 60 anos. A mortalidade anual em idosos, devido às quedas, apresenta um pico por volta dos 85 anos (MESQUITA, 2009).

Os traumas de baixa energia (quedas) representam um sério problema ao causar diminuição da capacidade funcional e institucionalização precoce do indivíduo, resultando em aumento na morbimortalidad. Essa situação é predominante entre idosos, devido a sarcopenia, disfunções nutricionais, presença de morbidades crônicas, diminuição da acuidade visual e dos reflexos e, principalmente, fragilidade óssea (NETO et al., 2011; AGUIAR et al., 2011).

Uma das principais consequências de queda nos idosos é a ocorrência de fraturas. Dentre elas, destaca-se a fratura do fêmur, com maior frequência de acometimento da porção proximal (colo do fêmur), transtrocanteriana e subtrocanteriana (MESQUITA et al., 2009). As fraturas de quadril são descritas como um importante fator de mortalidade em indivíduos acima de 65 anos (NEGRETE-CORONA, 2014).

O tratamento de escolha para a maioria dos pacientes é o cirúrgico, principalmente com artroplastia parcial do quadril, artroplastia total primária do quadril cimentada e tratamento cirúrgico de fratura em fêmur proximal. Porém, a realização de cirurgia nesse tipo de paciente pode gerar complicações sérias, com destaque para a ocorrência de infecções, principalmente em trato urinário e na ferida operatória, pneumonia e delirium (CUNHA et al, 2006), além da observação de elevada mortalidade no segmento após a realização do procedimento cirúrgico.

As causas para a elevada mortalidade em pacientes idosos com fratura da extremidade proximal do fêmur foram bem investigadas nos países desenvolvidos; no entanto, pouco se sabe sobre este assunto nos países em desenvolvimento (ARLIANI, 2011). 
Dessa forma, o objetivo da presente pesquisa foi avaliar os fatores de impacto na morbimortalidade de idosos com fraturas de quadril por fragilidade óssea assistidos em um centro de referência em Ortopedia de Brasília, Brasil.

\section{FUNDAMENTAÇÃO TEÓRICA}

De acordo com a Lei $n^{\circ} 10.741 / 2003$, de $1^{\circ}$ de outubro de 2003, o Estatuto do Idoso define idoso como o indivíduo com idade igual ou superior a 60 anos. Essa parcela da população está em um crescente aumento global, decorrente, sobretudo, de queda na taxa de mortalidade, da melhora na qualidade dos indicadores de saúde e da redução da taxa de fecundidade (CAMARANO, 2002).

O envelhecimento é definido como um processo progressivo, gradual e variável, caracterizado pela perda crescente de reserva funcional. Em consequência ocorrem alterações morfológicas, fisiológicas, bioquímicas e psicológicas, tornando o indivíduo mais propenso a adoecer, o que aumenta suas chances de morte. Essas mudanças que ocorrem no envelhecimento podem levar a limitações funcionais associadas a afecções agudas ou crônicas, tornando os idosos mais suscetíveis aos riscos ambientais, e consequentemente a ocorrência de quedas (MESQUITA, 2009).

A queda é o acidente que ocorre com maior frequência no idoso, sendo suas complicações a principal causa de morte naqueles com mais de 65 anos. Nesse sentido, as fraturas de colo de fêmur, em idosos, ocupam um papel de grande importância, tanto pela sua frequência, quanto pela sua gravidade, visto que levam ao aumento da dependência e da mortalidade de aproximadamente 50\%, em um ano (MESQUITA, 2009).

A diminuição da massa óssea que ocorre na osteoporose pós-menopausa (tipo I) afeta principalmente as mulheres com idades entre 50 e 70 anos. Nesse caso, o maior acometimento ocorre no osso trabecular, afetando as vértebras e o rádio distal. Na osteoporose senil ou tipo II, que ocorre após os 65-70 anos, a diminuição de massa óssea ocorre em consequência da diminuição da resposta hormonal e nutricional de osteoclastos e de osteoblastos devido à senilidade. Nessa faixa etária, a desmineralização óssea ocorre no osso trabecular e cortical, sendo o índice de fratura mais elevado na região cortical do quadril, tanto para mulheres quanto para homens (BELSKY et al., 2015)

Os principais determinantes esqueléticos do risco de fratura na pós-menopausa são a perda óssea e a destruição da arquitetura trabecular do osso. Quanto aos fatores extra- 
esqueléticos, a frequência e o tipo de queda e a adequação dos mecanismos de proteção neuromusculares têm maior impacto com a idade avançada (BORTOLON, 2010).

Nos países desenvolvidos sugere-se que alguns fatores pré-operatórios estão associados ao aumento da mortalidade em pacientes com fratura do quadril na admissão, como por exemplo: cor (não branca), idade, presença de demência, sexo masculino, comorbidades clínicas e delirium (MAJUMDAR, 2006).

Estudos ressaltam a alta prevalência de afecções crônicas prévias à fratura como complicador da recuperação, bem como o tempo pré-operatório como justificativa para pior prognóstico, atribuindo essa falha às dificuldades de acesso à cirurgia no serviço público de saúde nos locais avaliados (DINAMARCA-MONTECINOS et al., 2015; NEGRETECORONA, 2014). A sepse foi relatada como causa importante de mortalidade em uma pesquisa mexicana (NEGRETE-CORONA, 2014).

O tempo ideal entre o trauma e a cirurgia na fratura do fêmur proximal é bastante estudado e há bastante divergência entre os achados. Durante a formação de um grupo de trabalho, que ocorreu no Congresso Anual da Sociedade Internacional para o Reparo das Fraturas, o tratamento ideal foi preconizado como entre $24 / 48$ horas e em centro especializado (GOMES, 2014). No entanto, é controverso o tempo de espera para cirurgia como risco para o óbito. A literatura atual sugere não haver uma relação entre o tempo de espera cirúrgica e a mortalidade (ETXEBARRIA-FORONDA et al., 2013).

A maioria dos estudos sobre esse assunto foca, principalmente, a mortalidade, a qual é elevada na população idosa. No entanto, os estudos que avaliam a morbidade, ou seja, a perda de função pós-fratura são menos comuns. Por outro lado, os pacientes que sofrem fratura apresentam características próprias como menor massa óssea e maior limitação física medida pelas atividades da vida diária (AVD) e instrumentais da vida diária (AIVD), e capacidade de deambulação (WILLIAMS; JESTER, 2005).

Assim, devido ao crescente aumento da população idosa brasileira, muito se deve discutir sobre tais eventos incapacitantes nessa faixa etária. No decorrer do ciclo da vida podem acontecer situações que resultem em perda de autonomia e independência, sendo uma delas, a queda. Quando relacionadas ao idoso são consideradas de caráter relevante, pois podem leválos à incapacidade, injúria e morte. $\mathrm{O}$ custo social é proporcional à diminuição da autonomia e da independência ou a necessidade de institucionalização (MESQUITA, 2009).

Evidencia-se, pois, relação estreita entre funcionalidade e bem estar. É por este motivo que, do ponto de vista da saúde, a capacidade funcional surge como o conceito de saúde mais adequado para instrumentalizar e operacionalizar uma política contemporânea de atenção à 
saúde do idoso. Além disso, as políticas públicas direcionadas aos idosos evocam a promoção do envelhecimento ativo, que se relaciona à manutenção da autonomia e independência, preservando a funcionalidade e a qualidade de vida.

$\mathrm{O}$ atendimento de idosos frágeis, portanto, deve ser contínuo e ter como foco a avaliação e monitoramento da capacidade funcional, a avaliação e monitoramento dos medicamentos, a redução dos riscos de queda e o monitoramento do autocuidado e do trabalho dos cuidadores e da família.

\section{METODOLOGIA}

\subsection{Tipificação}

Tratou-se de pesquisa quantitativa, descritiva e analítica, que objetivou avaliar hipóteses de associações entre a exposição, suas características e o evento, ou seja, descrever as associações e correlações entre variáveis relacionadas a fraturas por queda em idosos e a mortalidade.

\subsection{Cenário da pesquisa}

Enfermaria de Ortopedia e Traumatologia do Hospital Regional do Paranoá, pertencente à Secretaria de Saúde do Distrito Federal. Inaugurado em 2002, o hospital é referência na área Ortopédica no Distrito Federal e possui serviço de Residencia Médica em Ortopedia e Traumatologia credenciada pela Sociedade Brasileira de Ortopedia e Traumatologia (SBOT). A Enfermaria possui 64 leitos ativos para internação, com taxa de ocupação média próxima a 97\%. O ano de inicio do estudo é compativel com o credenciamento do Hospital pela SBOT.

\subsection{Objetivo de estudo}

O motivo que impulsiona o estudo em questão é identificar o perfil e fatores clínicos dos pacientes acometidos por fraturas de quadril por fragilidade - decorrente de traumas de baixa energia - em pacientes acima de 60 anos no Distrito Federal previamente ao evento; identificar os fatores associados à internação hospitalar dos idosos com fratura, no pré, peri e pósoperatórios, e correlacioná-los ao evento mortalidade. Além disso, outras questões devem ser analisadas como identificar a taxa de mortalidade por fratura e os fatores que impactam na sobrevivência desses pacientes. 


\subsection{Delimitação e universo da amostra}

Para responder aos objetivos específicos foram avaliados todos os prontuários de pacientes maiores de 60 anos, com fraturas de quadril por trauma de baixa energia, devidamente registrada no momento de admissão em prontuário médico, residentes ou não no Distrito Federal, que internaram na enfermaria de Ortopedia do Hospital Regional do Paranoá, no período de janeiro de 2010 a janeiro de 2017.

Critérios de inclusão:

- Pacientes internados na enfermaria de Ortopedia do Hospital Regional do Paranoá, no período de janeiro de 2010 a janeiro de 2017.

- Pacientes cujo diagnóstico de queda da própria altura ou menos (trauma de baixa energia) devidamente registrados em prontuário no momento da admissão.

- Ter idade $\geq 60$ anos

Critérios de exclusão:

- Pacientes com fraturas patológicas por neoplasia óssea, mieloma múltiplo ou doença de Paget.

- Prontuários cujos registros de admissão estejam incompletos ou sem fundamentação que permitam identificar a causa do evento (queda da própria altura ou menos).

\subsection{Instrumento de coleta de dados}

Para identificação e descrição das variáveis, os dados foram coletados por meio do registro de admissão dos pacientes na enfermaria de Ortopedia, através do prontuário eletrônico Trak-care. Em seguida, cada prontuário clinico referente ao paciente-caso identificado foi analisado, individualmente e sequencialmente, onde foi feita a seleção das variáveis de interesse ao estudo e transcrição para um banco de dados $\left(\right.$ Excell $\left.^{\circledR}\right)$ para posterior análise estatística.

\subsection{Procedimentos metodológicos}

As análises estatísticas foram realizadas usando o programa SPSS (Statistical Package for the Social Sciences) versão 18.0. As variáveis quantitativas foram testadas para a distribuição normal com o teste de Shapiro-Wilk. Quando os dados foram considerados normais, foram utilizados os testes t de student. Para os dados que não puderem ter a hipótese de normalidade de dados aplicada, foi utilizada estatística não-paramétrica (teste de Mann-Whitney e Kruskal Wallis). Para análise da correlação entre duas variáveis quantitativas foi aplicado o coeficiente de correlação de Pearson ou Spearman. Para análise das variáveis qualitativas foi utilizado o 
teste Qui-Quadrado ou o teste Exato de Fisher. Valores de $\mathrm{p}<0,05$ foram considerados estatisticamente significativos.

Os pacientes foram estratificados em dois grupos (mortalidade e não mortalidade) e foi realizada uma regressão logística com ajuste para idade, com cada variável para estimar o risco relativo de associação entre essas e o evento mortalidade.

Além disso, foi realizada análise de sobrevivência (Kaplan-Meier) e a regressão de COX para avaliar os fatores que impactam na sobrevivência.

\section{RESULTADOS E DISCUSSÕES}

Os pacientes idosos são mais suscetíveis à fratura ou ao trauma cranioencefálico, sendo maior o risco para fraturas no fêmur e coluna vertebral. Esse grupo etário apresenta maior necessidade de acompanhamento ambulatorial e internação, uma vez que as lesões decorrentes de trauma em idosos podem requerer um longo período de tratamento, aumentando o número de complicações que levam a desfechos desfavoráveis, como cronicidade, incapacidade, invalidez e morte. (Cunha et al., 2008; Broska Junior et al., 2013). Dessa forma, ressalta-se a importância de alimentar os bancos de dados de forma completa, para que seja possível estabelecer medidas para reduzir as consequências da fragilidade óssea.

Em estudo brasileiro, Bortolon et al. (2011) observaram que o percentual de internações de idosos com diagnóstico principal de fratura de fêmur foi baixo em relação a outros países, o que os autores atribuíram às possíveis subnotificações de casos relacionados que ficam sem atendimento. Além disso, Broska Junior et al. (2013) ressaltam que, em relação aos traumas em idosos, a queda da própria altura precede os acidentes de trânsito (atropelamentos), o que também pode justificar a observação de Bortolon et al. (2011).

Para a presente pesquisa, foram avaliados todos os prontuários de pacientes maiores de 60 anos, com fraturas de quadril por trauma de baixa energia, totalizando 347 sujeitos. A partir da análise dos dados obtidos com a revisão dos prontuários, percebeu-se a predominância de fraturas de fêmur decorrentes de traumas de baixa energia (quedas) entre as mulheres $(n=229)$, em conformidade com o observado em outros estudos (Arliani et al., 2011; BORTOLON et al., 2011; Broska Junior et al. 2013; CUNHA et al., 2008; FRANCO et al., 2015; SOUZA et al., 2007).

O desfecho mortaliade foi identificado em 76 pacientes (21,8\%), sendo 52,68\% destes do gênero feminino $(p=0,544)$. Em relação à idade, houve maior mortalidade $(p<0,005)$ entre os idosos com média de idade de 84,7 $\pm 1,0$ anos, o que também foi verificado por Cunha et al. (2008) e Arliani et al. (2011). Nos estudos de Dinamarca-Montecinos et al. (2015) e Ojeda et 
al. (2013), ressalta-se que mulheres são mais afetadas pelas fraturas de quadril em comparação aos homens, e no estudo de Negrete-Corona (2014), em relação ao sexo, foi verificada maior mortalidade em mulheres idosas com fraturas de quadril.

O tratamento de escolha para a maioria dos pacientes com fratura de fêmur é o cirúrgico. Porém, a realização de cirurgia nesse tipo de paciente pode gerar complicações sérias, com destaque para a ocorrência de infecções, principalmente em trato urinário e na ferida operatória, pneumonia e delirium (CUNHA et al., 2006), o que propicia elevada mortalidade no segmento após a realização do procedimento cirúrgico. A tabela 1 apresenta os possíveis fatores preditores de mortalidade intra-hospitalar identificados na amostra em relação ao desfecho do paciente.

Tabela 1. Fatores preditores de mortalidade intra-hospitalar em pacientes idosos com fratura de fêmur.

\begin{tabular}{|c|c|c|c|c|c|}
\hline \multirow{2}{*}{ Fatores preditores } & & \multicolumn{2}{|c|}{ Mortalidade } & \multirow{2}{*}{$\begin{array}{l}p \text { - value } \\
<0,005^{*}\end{array}$} & \multirow{2}{*}{ OR } \\
\hline & & $\operatorname{Sim}(n=76)$ & Não $(n=271)$ & & \\
\hline \multirow{2}{*}{ Infecção } & Sim & $61(81,3 \%)$ & $57(21,7 \%)$ & \multirow{2}{*}{$0,000^{*}$} & \multirow{2}{*}{5.7} \\
\hline & Não & $14(18,7 \%)$ & $206(78,3 \%)$ & & \\
\hline \multirow{2}{*}{ Infecção pulmonar } & Sim & $49(64,5 \%)$ & $21(7,8 \%)$ & \multirow{2}{*}{$0,000^{*}$} & \multirow{2}{*}{7.38} \\
\hline & Não & $27(35,5 \%)$ & $249(92,2 \%)$ & & \\
\hline \multirow{2}{*}{ Infecção urinária } & Sim & $22(28,9 \%)$ & $22(8,2 \%)$ & \multirow{2}{*}{$0,000^{*}$} & \\
\hline & Não & $54(71,1 \%)$ & $247(91,8 \%)$ & & \\
\hline \multirow{2}{*}{$\begin{array}{l}\text { Hospitalização em } \\
\text { UTI }\end{array}$} & Sim & $46(62,2 \%)$ & $129(47,6 \%)$ & \multirow{2}{*}{0,026} & \multirow{2}{*}{1,10} \\
\hline & Não & $28(37,8 \%)$ & $142(52,4 \%)$ & & \\
\hline \multirow{2}{*}{ Hipertensão } & Sim & $69(92 \%)$ & $189(69,7 \%)$ & \multirow{2}{*}{$0,001 *$} & \multirow{2}{*}{4,05} \\
\hline & Não & $6(8 \%)$ & $82(30,3 \%)$ & & \\
\hline \multirow{2}{*}{ Diabetes Mellitus } & Sim & $36(48 \%)$ & $86(31,7 \%)$ & \multirow[b]{2}{*}{0,009} & \\
\hline & Não & $39(52,0 \%)$ & $185(68,3 \%)$ & & \\
\hline \multirow{2}{*}{$\begin{array}{l}\text { Comprometimento } \\
\text { Cognitivo/ } \\
\text { Depressão }\end{array}$} & Sim & $33(44,6 \%)$ & $49(18,1 \%)$ & \multirow[b]{2}{*}{0,000} & \multirow[b]{2}{*}{1,9} \\
\hline & Não & $41(55,4 \%)$ & $222(81,9 \%)$ & & \\
\hline
\end{tabular}




\begin{tabular}{|c|c|c|c|c|}
\hline \multirow{3}{*}{ Detsky (1997) } & Baixo (I) & $3(4,0 \%)$ & $47(17,8 \%)$ & \multirow{3}{*}{0,000} \\
\hline & Moderado (II) & $21(28,0 \%)$ & $147(55,7 \%)$ & \\
\hline & Alto ( III ) & $51(68,0 \%)$ & $70(26,5 \%)$ & \\
\hline
\end{tabular}

Sabe-se que quanto maior o número de comorbidades no paciente idoso fraturado, maior é a chance de evolução para óbito (SOUZA et al., 2007; ARLIANI et al. 2011; AGUIAR et al. 2011; FRANCO et al., 2015). Acerca dos fatores relacionados ao desfecho mortalidade, no presente trabalho destacaram-se a presença de Diabetes Mellitus em 48\% dos pacientes, apesar de esse não ter sido um fator preditor de mortalidade, e a classificação Destsky (1997) III (68\% dos pacientes).

Os fatores de risco associados à mortalidade identificados na amostra foram: Prejuízo Cognitivo/Depressão $(44,6 \%)$ [OR=1,9], Hipertensão (HAS) $(92 \%)$ [OR=4,05], Infecção (35\%) [OR=5,7] e Infecção Respiratória (64,5\% ) [OR=7,38].

Aguiar et al. (2011) e Pan et al. (2018) destacaram o papel da depressão como determinante de mortalidade, o que também foi observado neste estudo. Apesar de o mecanismo exato de como a depressão contribui com a fratura de fêmur ser desconhecido, acredita-se que a baixa densidade mineral óssea encontrada em pacientes deprimidos possa influenciar a ocorrência das fraturas.

A hipertensão arterial demonstrou-se um fator preditivo de maior mortalidade. O uso de anti-hipertensivos, particularmente de diuréticos de alça e IECA, são associados a um risco maior de fraturas de quadril (RUTHS et al., 2015). Além disso, afecções cardiovasculares em geral tem sido descritas como um dos principais fatores associados à mortalidade precoce de pacientes com fraturas de fêmur (CHOI et al., 2018).

Além disso, a literatura relata a presença de infecção no período pós operatório de fraturas de fêmur em torno de 30\% (BORTOLON et al., 2011), semelhante ao encontrado na presente amostra. Henderson et al. (2015) ressalta que contrair infecção respiratória após fratura de quadril aumenta significativamente a mortalidade em 1 ano. Na presente amostra verificouse que a maioria dos pacientes cujo desfecho não foi a morte $(n=271)$ não tinham infecção respiratória como comorbidade $(92,2 \%)$. Ademais, a maior parte dos pacientes que vieram a óbito tiveram esse tipo de complicação $(64,5 \%)$.

A tabela 2 informa os fatores preditores de mortalidade relacionados à realização ou não de procedimento cirúrgico, ao tipo cirurgia e ao tempo decorrido durante a internação. 
Tabela 2. Fatores preditores de mortalidade relacionados à cirurgia e internação.

\begin{tabular}{|c|c|c|c|c|c|}
\hline \multirow{2}{*}{\multicolumn{2}{|c|}{ Fatores preditores }} & \multicolumn{2}{|c|}{ Mortalidade } & \multirow{2}{*}{$\begin{array}{c}p \text {-value } \\
(p<0,005)\end{array}$} & \multirow{2}{*}{ OR } \\
\hline & & Sim & Não & & \\
\hline \multicolumn{2}{|c|}{ Dias de hospitalização } & $30,9 \pm 2,5$ & $22,2 \pm 1,1$ & 0,000 & \\
\hline \multicolumn{2}{|c|}{ Dias até a cirurgia } & $17,9 \pm 1,7$ & $16,8 \pm 0,8$ & 0,353 & \\
\hline \multicolumn{2}{|c|}{ Dias de pós operatório } & $19,6 \pm 3,4$ & $6, \pm 0,8$ & 0,000 & \\
\hline & & $\operatorname{Sim}(n=76)$ & Não $(\mathrm{n}=271)$ & & \\
\hline \multirow{2}{*}{$\begin{array}{l}\text { Cirurgia } \\
\text { realizada }\end{array}$} & Sim & $43(56,6 \%)$ & $220(81,5 \%)$ & \multirow{2}{*}{$0,000^{*}$} & \multirow{2}{*}{2.10} \\
\hline & Não & $33(43,4 \%)$ & $50(18,5 \%)$ & & \\
\hline \multirow{2}{*}{ Cirurgias } & Osteossíntese & $25(59,5 \%)$ & $123(55,4 \%)$ & \multirow{2}{*}{0,622} & \multirow{2}{*}{2.10} \\
\hline & Artroplastia & $17(40,5 \%)$ & $99(44,6 \%)$ & & \\
\hline
\end{tabular}

Não houve diferença significativa no tempo até a cirurgia em relação à mortalidade dos pacientes, divergentemente do encontrado por Cunha et al. (2008). O número de dias de internação $(30,9 \pm 2,5)$, o tempo de pós-operatório $(19,6 \pm 3,4)$ e a internação em UTI $(13,9 \pm$ $2,4)[\mathrm{OR}=1,10]$ foram significativos na identificação de fatores associados à mortalidade dos pacientes que compuseram a amostra.

O tempo médio de internações de idosos por fratura de fêmur em 2008 relatado no Brasil foi de um a sete dias em mais de metade dos casos (BORTOLON et al., 2011). Arliani et al. (2011) encontraram um tempo médio de 10,7 dias, ressaltando que os pacientes com tempo de internação superior a sete dias apresentaram maior mortalidade. Franco et al. (2015) não encontraram associação entre o tempo de internação e o desfecho mortalidade intra-hospitalar.

Os dados apresentados na literatura indicam um número bastante inferior ao encontrado presente pesquisa. Ao mesmo tempo em que a alta precoce pode reduzir o tempo de exposição a agentes infecciosos intra-hospitalares e suas decorrentes complicações sobretudo a morte (AGUIAR et al. 2011), com o tempo curto de internação interrompe-se o cuidado e o segmento do paciente em recuperação, não sendo possível determinar a sobrevida após a intervenção decorrente da fratura.

Semelhante aos trabalhos de Arliani et al. (2011), Ribeiro et al. (2014) e Franco et al. (2015), não houve associação neste estudo entre o tempo até a cirurgia e a mortalidade, diferente do encontrado por Souza et al. (2007). A realização de intervenção cirúrgica foi associada à 
mortalidade $(56,6 \%)$ [OR $=2,10]$, resultado divergente do obtido por Franco et al. (2015), em que a taxa de sobrevida foi significativamente maior nos indivíduos que foram submetidos ao procedimento cirúrgico. Considerando apenas os pacientes operados e o tempo de pósoperatório, verificou-se que a média de sobrevida foi de 39,2 dias.

Os pacientes submetidos à osteossíntese $(56,4 \%)$ tiveram um tempo de sobrevida menor que à artroplastia $(43,6 \%)$ [HR = 2,1 IC 95\%: 1,03-4,41]. Franco et al. (2015) e Ribeiro et al. (2014) concluíram que o tipo de abordagem cirúrgica não teve relação significativa com o tempo de recuperação nem com o óbito. No entanto, Paula et al. (2015) ressalta que as artroplastias representaram um risco mais elevado de readmissão, quando comparadas à osteossíntese, o que não foi avaliado no presente trabalho.

\section{CONCLUSÃO}

A partir da análise e da discussão das informações obtidas com a presente pesquisa depreende-se que atenção especial deve ser dada aos idosos com fratura por fragilidade óssea. $\mathrm{Na}$ amostra analisada, foi possível observar que pacientes idosos com condições de saúde frágil e diversas comorbidades permanecem por mais tempo em internação hospitalar, o que aumenta o risco de mortalidade.

A ocorrência de infecção e a presença de hipertensão arterial foram as principais comorbidades identificadas para a maior mortalidade. O tipo de fratura e o risco cirúrgico também determinaram a opção cirúrgica e o desfecho analisado. A fratura do colo do fêmur é, portanto, um importante indicador do processo de envelhecimento e possui íntima relação com a longevidade de pacientes idosos.

\section{REFERENCIAS:}

AGUIAR, Fernanda de Jesus; NEMER, Danielle Saad e LEME, Luiz Eugênio Garcez. Estado nutricional e evolução de cirurgias ortopédicas de urgência em idosos. Acta Ortop Bras. v. 19, n. 5, p. 293-8, 2011.

ARLIANI, Gustavo Gonçalves; ASTUR, Diego da Costa; LINHARES, Glauber Kazuo; BALBACHEVSKY, Daniel; FERNANDES Hélio Jorge Alvachian e REIS Fernando Baldy dos. Correlação entre tempo para o tratamento cirúrgico e mortalidade em pacientes idosos com fratura da extremidade proximal do fêmur. Rev. bras. ortop. v. 46, n. 2, p. 189-194, Apr 2011. 
BELSKY, DW et. al. Quantification of biological aging in young adults. Proc Natl Acad Sci USA. v. 112, n. 30, p 4104-10, 2015.

BORTOLON, PC. Envelhecimento e osteoporose senil: descrição do atendimento hospitalar para fratura de fêmur no sus. [Dissertação de mestrado] Escola Nacional de Saúde Pública, Fundação Oswaldo Cruz. Rio de Janeiro, 2010.

BORTOLON, Paula Chagas; DE ANDRADE, Carla Lourenço Tavares e DE ANDRADE, Carlos Augusto Ferreira. Internações do sus para fratura osteoporótica de fêmur em idosos. Cad. Saúde Pública, v. 27, n. 4, p. 733-742, abr 2011.

BROSKA JÚNIOR, César Augusto; DE FOLCHINI, Augusto Bernardo e DE RUEDIGER, Ricardo Rydygier. Estudo comparativo entre o trauma em idosos e não idosos atendidos em um Hospital Universitário de Curitiba. Rev. Col. Bras. Cir. v. 40, n. 4, p. 281-286, 2014.

CHOI, HG; LEE, YB; RHYU, SH; KWON, BC e LEE, JK. Mortality and cause of death postoperatively in patients with a hip fracture - a national cohort longitudinal follow-up study. Bone Joint J, v. 100-B, p. 436-42, 2018.

CUNHA, Priscilla Tatiane Silveira; ARTIFON, Aline Nurchis; LIMA, Danielle Pessoa; MARQUES, Wanessa Vieira; RAHAL, Miguel Antonio; RIBEIRO, Ricardo R; KITADAI, Fábio Takashi. Fratura de quadril em idosos: tempo de abordagem cirúrgica e sua associação quanto a delirium e infecção. Acta Ortop Bras, v. 16, n. 3, p. 173-176, 2006.

DINAMARCA-MONTECINOS, José Luis; AMÉSTICA-LAZCANO, Gedeón; RUBIOHERRERAA, Ramona; CARRASCO-BUVINIC, Alejandro e VÁSQUEZ, Alejandra. Características epidemiológicas y clínicas de las fracturas de cadera en adultos mayores en un hospital público chileno. Rev Med Chile, v. 143, p. 1552-1559, 2015.

ETXEBARRIA-FORONDA, J; MAR, A. ARROSPIDE; EGUINO, J. Cost and mortality associated to the surgical delay of patients with a hip fracture. Rev Esp Salud Publica, v. 87, n. 6, p. 639-649, 2013. 
FRANCO, Léo Graciolli; KINDERMANN, Amanda Loffi; TRAMUJAS, Lucas e KOCK, Kelser de Souza. Fatores associados à mortalidade em idosos hospitalizados por fraturas de fêmur. Rev bras ortop. v. 51, n. 5, p. 509-514, 2015.

GOMES, ECC et al. Factors associated with the danger of accidental falls among institutionalized elderly individuals: an integrative review. Ciênc. saúde coletiva v. 19, n. 8, p. 3543-3551, 2014.

MESQUITA, GV. Morbimortalidade em idosos por fratura proximal do fêmur. Texto Contexto Enferm, v. 18, n. 1, p. 67-73, Jan-Mar 2009.

NEGRETE-CORONA, J; ALVARADO-SORIANO, JC; REYES-SANTIAGO, LA. Fractura de cadera como factor de riesgo en la mortalidad en pacientes mayores de 65 años. Estudio de casos y controles. Acta Ortopédica Mexicana, v. 28, n. 6, p. 352-362, nov-dez 2014.

NETO, José Soares Hungria; DIAS, Caio Roncon e ALEMIDA, José Daniel Bula. Epidemiological characteristics and causes of proximal femoral fractures among the elderly. Rev Bras Ortop, v. 46, n. 6, p. 660-67, 2011.

OJEDA, Dagoberto; GAZABATT, Florence; CISTERNAS, Patricia; FOLCH, Francisca e DEMPSTER, Christopher. Sobrevida postoperatoria en pacientes nonagenarios. Estudio pronóstico en cohorte retrospectiva 2001-2010, Clínica Dávila. Rev Med Chile, v.141, p. 3440, 2013.

PAN, C; HU, L; LU, T; TU, M; SHEN, C E CHEN, Z. Risk of hip fractures in patients with depressive disorders: A nationwide, population-based, retrospective, cohort study. PLoS One, v. 13, n. $4,2018$.

RIBEIRO, Tiango Aguiar; PREMAOR, Melissa Orlandin; LARANGEIRA, João Alberto; BRITO, Luiz Giulian; LUFT, Michel; GUTERRES, Leonardo Waihrich e MONTICIELO, Odirlei André. Predictors of hip fracture mortality at a general hospital in South Brazil: an unacceptable surgical delay. Clinics, v. 69, n. 4, p. 253-258, 2014. 
RUTHS, S; BAKKEN, MS; RANHOFF, AH; HUNSKAAR, S; ENGESÆTER, LB E ENGELAND, A. Risk of hip fracture among older people using antihypertensive drugs: a nationwide cohort study. BMC Geriatr, v. 15, p. 153, 2018.

SOUZA, Rômulo Cristovão; PINHEIRO, Rejane Sobrino; COELI, Cláudia Medina; CAMARGO Jr., Kenneth Rochel e DE TORRES, Tânia Z Guillén. Aplicação de medidas de ajuste de risco para a mortalidade após fratura proximal de fêmur. Rev Saúde Pública, v. 41, n. 4, p. 625-31, 2007.

STOLNICK, B. Para que a primeira fratura seja a ultima. RBO, v. 51, p. 121,122, mar-abr, 2016.

WILLIAMS, A e JESTER, R. Delayed surgical fixation of fractured hips in older people: impact on mortality. J Adv Nurs, v. 52, n. 1, p. 63-9, 2005. 